\title{
Dynamical Coulomb blockade of thermal transport
}

\author{
Guillem Rosselló, ${ }^{1}$ Rosa López, ${ }^{1}$ and Rafael Sánchez ${ }^{2}$ \\ ${ }^{1}$ Institut de Física Interdisciplinar i de Sistemes Complexos (CSIC-UIB), E-07122 Palma de Mallorca, Spain \\ ${ }^{2}$ Instituto Gregorio Millán, Universidad Carlos III de Madrid, E-28911 Leganés, Madrid, Spain \\ (Received 13 January 2017; revised manuscript received 8 May 2017; published 2 June 2017)
}

\begin{abstract}
The role of energy exchange between a quantum system and its environment is investigated from the perspective of the Onsager conductance matrix. We consider the thermoelectric linear transport of an interacting quantum dot coupled to two terminals under the influence of an electrical potential and a thermal bias. We implement in our model the effect of coupling to electromagnetic environmental modes created by nearby electrons within the $P(E)$ theory of dynamical Coulomb blockade. Our findings relate the lack of some symmetries among the Onsager matrix coefficients to an enhancement of the efficiency at maximum power and the occurrence of the heat rectification phenomenon.
\end{abstract}

DOI: 10.1103/PhysRevB.95.235404

\section{INTRODUCTION}

The properties of electronic heat transport in nanostructures have recently attracted the attention of the scientific community for different reasons [1-3]. On the one hand, the onset of quantum effects in the mesoscopic regime opens the way to the investigation of the impact of quantum mechanics on thermodynamics [4]. In particular, heat engines based on purely quantum-mechanical effects have been recently proposed [5-10]. Complementary to this, there has been spectacular progress in the field of quantum thermoelectrics from both the theoretical and experimental sides. Exciting proposals like nanoprobe thermometers [1,11], energy-harvesting devices [12-16], refrigerators [17-21], heat diodes [22], rectifiers [23-26], transistors [27,28], and multiterminal heat engines [29-32], among others, have come up in recent years.

In this respect, quantum dots [33-43] have a prominent role for being good energy filters that improve the thermoelectric efficiency $[44,45]$. The presence of strong interactions introduces the Coulomb blockade regime where transport can be controlled at the level of single-electron tunneling events [46,47]. Different functionalities such as heat engines [14], pumps [48-50], and diodes [22] can be defined that use these properties.

Currents are small in nanostructures and are hence sensible to fluctuations. The question arises of how the system behavior is influenced by a noisy environment. On the one hand, it leads to dephasing and decoherence, which are detrimental to quantum coherent processes. This is, however, not necessarily a drawback [51,52]. On the other hand, they may lead to inelastic transitions which can contribute to the engine performance by injecting or releasing energy in the conductor [53-55]. Indeed, nonlocal thermoelectric engines exist that use an environment as a heat source in an otherwise equilibrated conductor. The nature of the environment can be either fermionic $[30,56]$ or bosonic [29,57]. It can also consist of transport fluctuations in a Coulomb coupled conductor [58-63] or be due to quantum fluctuations in an electromagnetic environment $[64,65]$. This last effect has been observed in the form of the dynamical Coulomb blockade of charge currents [66-70].

The linear response of a two-terminal nanodevice is defined on the grounds of the Onsager-Casimir relations [71-73]. The Onsager coefficients $\mathcal{O}_{i j}=\partial \mathcal{I}_{i} / \partial \mathcal{A}_{j}$ relate the charge and heat fluxes $\mathcal{I}_{i} \equiv\left\{I_{i}^{e}, I_{i}^{h}\right\}$ in terminal $i$ to the thermodynamic affinities $\mathcal{A}_{j}$. These can be due to electric or thermal gradients. Briefly, the coefficients $\mathcal{O}_{i j}$ can be collected into the so-called Onsager conductance matrix, which is a symmetric and positively semidefined matrix [74]. Derived from the principle of microreversibility, Onsager reciprocity relations identify nondiagonal coefficients $\mathcal{O}_{i j}$, e.g., Seebeck and Peltier responses. Notoriously, such relations are also satisfied for quantum systems independently of the presence of interactions. For quantum systems in which phase coherence is preserved, additional relations for the Onsager coefficients are obtained from the unitarity of the electron dynamics $[75,76]$, giving rise to highly symmetric Onsager matrices. However, the interaction with an environment introduces energy dissipation which prevents the dynamics from being unitary. In particular some relations among the thermoelectric coefficients are no longer satisfied [77,78]. The microscopic origin of such asymmetries has so far not been discussed.

In this work we explore this issue by using a microscopic model for the coupling of a mesoscopic system to a dynamical environment introduced as a circuit impedance [79]. We consider the simplest situation of a single-level quantum dot tunnel coupled to two terminals, $\mathrm{L}$ and $\mathrm{R}$, via tunneling barriers (see Fig. 1). Electrons tunneling through each of the barriers perceive a different environment, as it also includes the other barrier. This results in photon-assisted tunneling events which
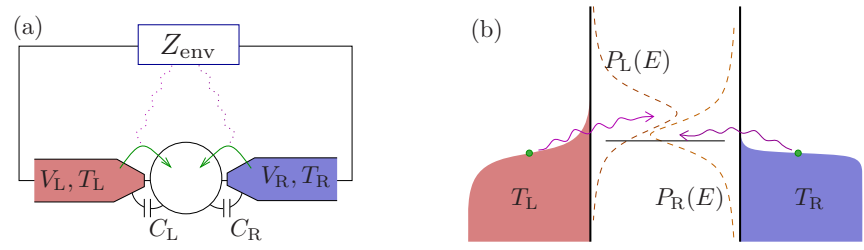

FIG. 1. (a) Schematic of a two-terminal single-level quantum dot device in the presence of an electromagnetic environment described by an external impedance $Z_{\text {env }}(\omega)$. Each terminal is electrically and thermally biased with $V_{\mathrm{L}}, T_{\mathrm{L}}=T_{0}+\Delta T_{\mathrm{L}}$ (left contact) and $V_{\mathrm{R}}, T_{\mathrm{R}}=$ $T_{0}+\Delta T_{\mathrm{R}}$ (right contact). The capacitance of each junction $C_{i}$, with $i=L, R$, determines the dynamical coupling to the environment. (b) Inelastic tunneling into the quantum dot is then described by a distribution $P_{i}(E)$ which is different for each barrier. 
on top of becoming inelastic, introduce left-right asymmetric rates. As we show below, the occurrence of Onsager matrix asymmetries is due to a combination of these two effects. It leads to responses that depend not only on the global temperature gradient $\Delta T=T_{\mathrm{L}}-T_{\mathrm{R}}$ but rather on how it is distributed with respect to some reference temperature $T_{0}$ in the two leads, $\Delta T_{l}=T_{l}-T_{0}$. It affects the thermoelectric response, and most particularly, it introduces an apparent thermal rectification in the linear regime.

The remainder of this paper is organized as follows. In Sec. II our model is described. Results for the single- and double-occupation regimes are presented in Secs. III and IV, respectively, with conclusions discussed in Sec. V.

\section{THEORETICAL MODEL}

We consider a two-terminal interacting conductor as illustrated in Fig. 1. We use a spinful single-level quantum dot described by four states, $|0\rangle,|\mathrm{u}\rangle,|\mathrm{d}\rangle$, and $|2\rangle$. They correspond to an empty dot $(|0\rangle)$, a singly occupied dot with either spin up $(|\mathrm{u}\rangle)$ or down $(|\mathrm{d}\rangle)$ polarization, and the doubly occupied dot state $(|2\rangle)$. Our transport description is restricted to the sequential tunneling regime for which $h \Gamma \ll k_{B} T_{0}$, where $T_{0}$ is the base temperature. In this paper, $T_{0}$ will correspond to the temperature of the environment. In this regime, transport events are predominantly of the first order in the tunneling coupling $\Gamma$. To properly account for Coulomb interactions we employ the electrostatic model schematically illustrated in Fig. 1. In such a model, the electrostatic charging energy is described with two capacitances, $C_{\mathrm{L}}$ and $C_{\mathrm{R}}$. The dynamics of the system is modeled by the time evolution of the occupation probabilities $p \equiv$ $\left\{p_{0}, p_{\mathrm{u}}, p_{\mathrm{d}}, p_{2}\right\}$ described by the following master equation:

$$
\begin{aligned}
\frac{d p_{0}}{d t} & =\sum_{j}\left[-2 \Gamma_{\mathrm{j}, 0}^{+} p_{0}+\Gamma_{j, 0}^{-}\left(p_{\mathrm{u}}+p_{\mathrm{d}}\right)\right], \\
\frac{d p_{\sigma}}{d t} & \left.=\sum_{j}\left[-\left(\Gamma_{\mathrm{j}, 0}^{-}+\Gamma_{\mathrm{j}, 1}^{+}\right) p_{\sigma}+\Gamma_{j, 0}^{+} p_{0}+\Gamma_{j, 1}^{-} p_{2}\right)\right], \\
\frac{d p_{2}}{d t} & =\sum_{j}\left[-2 \Gamma_{\mathrm{j}, 1}^{-} p_{2}+\Gamma_{j, 1}^{+}\left(p_{\mathrm{u}}+p_{\mathrm{d}}\right)\right] .
\end{aligned}
$$

The transition rates $\Gamma_{j, s}^{ \pm}$for electrons tunneling in (+) or out $(-)$ of the dot through contact $j=\mathrm{L}, \mathrm{R}$ are given below. We do not consider a magnetic field, so they do not depend on spin. They depend on the electrochemical potential $\mu_{s}$ when the dot is empty, $s=0$, or singly occupied, $s=1$. A simple electrostatic model [80] yields

$$
\mu_{s}=\epsilon_{\mathrm{d}}+\frac{e^{2}(1+2 s)}{2 C}+e\left(\kappa_{\mathrm{L}} V_{\mathrm{L}}+\kappa_{\mathrm{R}} V_{\mathrm{R}}\right),
$$

where $e$ is the electron charge, $\epsilon_{\mathrm{d}}$ is the bare energy level of the quantum dot, $C=C_{\mathrm{L}}+C_{\mathrm{R}}$ is its total capacitance, and $\kappa_{j}=1-C_{j} / C$, with $j=\mathrm{L}, \mathrm{R}$ (see Fig. 1 ).

\section{A. Tunneling rates}

Tunneling events are frequently affected by fluctuations of the electromagnetic environment [70]. To fully account for such quantum fluctuations we adopt the $P(E)$ theory [70,79] of dynamical Coulomb blockade, recently revisited to consider heat fluxes $[64,81]$. The spirit of the $P(E)$ theory relies on the fact that individual tunneling events involve energy exchange processes. The Dirac $\delta$ accounting for energy conservation in the (Fermi's golden rule) tunneling rates is relaxed into a broadened distribution $P(E)$. More specifically, for a double junction it reads

$$
P_{j}(E)=\frac{1}{2 \pi \hbar} \int d t \exp \left(\kappa_{i}^{2} J(t)+\frac{i}{\hbar} E t\right)
$$

where the function

$$
J(t)=\frac{2 h}{e^{2}} \int_{0}^{\infty} \frac{d \omega}{\omega} \operatorname{Re}[\tilde{Z}(\omega)] c\left(\omega, T_{0}\right)
$$

contains all the information of the environment fluctuations, with [79]

$$
c\left(\omega, T_{0}\right)=\operatorname{coth}\left(\frac{\hbar \omega}{2 k_{\mathrm{B}} T_{0}}\right)[\cos (\omega t-1)-i \sin \omega t] .
$$

If we consider a pure resistive or Ohmic environment, we have

$$
Z_{\text {env }}(\omega)=R \gg R_{\mathrm{q}}=h / 2 e^{2} .
$$

This situation corresponds to the case where the electron tunnel may easily excite many electromagnetic modes. Thus, the total impedance seen by the external circuit is

$$
\tilde{Z}(\omega)=\left[i \omega C_{\mathrm{eff}}+Z_{\mathrm{env}}(\omega)\right]^{-1},
$$

with the effective capacitance of the quantum $\operatorname{dot} C_{\mathrm{eff}}^{-1}=$ $C_{\mathrm{L}}^{-1}+C_{\mathrm{R}}^{-1}$. Semiconductor quantum dots in the sequential tunneling regime are typically affected by a high-impedance environment $[82,83]$. Under these considerations one obtains a Gaussian distribution:

$$
P_{j}(E)=\frac{1}{\left(4 \pi \kappa_{j}^{2} E_{C} k_{B} T_{0}\right)^{1 / 2}} e^{-\frac{\left(E-\kappa_{j}^{2} E_{C}\right)^{2}}{4 \kappa_{j}^{2} E_{C} k_{\mathrm{B}} T_{0}}},
$$

where $E_{C}=q^{2} \kappa_{\mathrm{L}} \kappa_{\mathrm{R}} / 2 C$. Remarkably, asymmetries in the system capacitances translate in the $P_{j}(E)$ functions having different means $\kappa_{j}^{2} E_{C}$ and variances $2 \kappa_{j}^{2} E_{C} k_{B} T_{0}$. They modify the transition-rate expressions according to

$$
\Gamma_{j, s}^{ \pm}=\Gamma_{j} \int d E f^{ \pm}\left(E-e V_{j}, T_{j}\right) P_{j}\left(E-\mu_{s}\right),
$$

where $f^{+}(E, T)=1 /\left[1+e^{E /\left(k_{\mathrm{B}} T\right)}\right]$ is the Fermi function and $f^{-}=1-f^{+}$. The quantum dot tunneling rates can be left-right asymmetric either for having barriers with different transparencies, $\Gamma_{\mathrm{L}} \neq \Gamma_{\mathrm{R}}$, or for $P_{\mathrm{L}}(E) \neq P_{\mathrm{R}}(E)$. We emphasize that having different $P_{j}(E)$ introduces an implicit asymmetry in the energy exchanged by the electrons with the environment in the tunneling processes. As we discuss below, the impact in the system response shows up in the thermal transport coefficients.

Finally, the charge and heat currents through contact $j$ are calculated through

$$
\begin{aligned}
& I_{j}^{\mathrm{e}}=e\left[\sum_{\sigma=\mathrm{u}, \mathrm{d}}\left(\Gamma_{j, 0}^{-}-\Gamma_{j, 1}^{+}\right) p_{\sigma}-2 \Gamma_{j, 0}^{+} p_{0}+2 \Gamma_{j, 1}^{-} p_{2}\right], \\
& I_{j}^{\mathrm{h}}=\sum_{\sigma=\mathrm{u}, \mathrm{d}}\left(\gamma_{j, 0}^{-}-\gamma_{j, 1}^{+}\right) p_{\sigma}-2 \gamma_{j, 0}^{+} p_{0}+2 \gamma_{j, 1}^{-} p_{2},
\end{aligned}
$$


where the transition rates for the heat current in Eq. (10) are given by

$$
\gamma_{j, s}^{ \pm}=\Gamma_{j} \int d E\left(E-e V_{j}\right) f^{ \pm}\left(E-e V_{j}\right) P_{j}\left(E-\mu_{s}\right) .
$$

These rates take into account the heat transported in each particle transition. Charge conservation ensures $I_{\mathrm{R}}^{\mathrm{e}}=-I_{\mathrm{L}}^{\mathrm{e}}$.

\section{B. Linear regime}

By linearizing the electrical, $I_{i}^{\mathrm{e}}$, and heat, $I_{i}^{\mathrm{h}}$, currents at the $i$ th reservoir in response to the applied thermodynamical forces $\left\{V_{j}, \Delta T_{j}\right\}$,

$$
\begin{aligned}
& I_{i}^{\mathrm{e}}=\sum_{j}\left(G_{i j} V_{j}+L_{i j} \Delta T_{j}\right), \\
& I_{i}^{\mathrm{h}}=\sum_{j}\left(M_{i j} V_{j}+K_{i j} \Delta T_{j}\right),
\end{aligned}
$$

we obtain the four conductance matrices that compose the Onsager matrix. Onsager-Casimir reciprocity relations dictate $G_{i j}=G_{j i}, K_{i j}=K_{j i}$, and $L_{i j}=e M_{j i} / T_{0}$. Additional relations imposed on the cross conductances $\left(L_{i j}=L_{j i}\right)$ arise in the case where transport occurs elastically.

In our setup, the tunneling rates in Eq. (9) describe inelastic processes. Hence, they introduce energy exchange with the environment. The energy of the two-terminal system is hence not conserved, $I_{\mathrm{L}}^{\mathrm{h}}+I_{\mathrm{R}}^{\mathrm{h}} \neq 0$. In the following we analyze the effect of inelasticity on the thermal coefficients, namely, $L_{i j}$ (or $M_{j i}$ ) and $K_{i j}$.

\section{Thermal coefficients}

Thermal rectification in an isoelectric $\left(V=V_{\mathrm{R}}-V_{\mathrm{L}}=0\right)$ two-terminal conductor occurs when the heat current becomes asymmetric on the reversal of the temperature gradient. It has been discussed that this is not possible in the linear regime for the heat current across the system, even in the presence of an environment or a thermal bath [54]. One has to take into account that energy is dissipated into the environment at the nanostructure. However, in an experiment this quantity is not easy to detect. One would rather measure the heat current at each terminal.

In this case, the measured heat current might be L-R asymmetric with the reversal of $\Delta T$, i.e.,

$$
\delta I_{\mathrm{LR}}^{\mathrm{h}}=I_{\mathrm{L}}^{\mathrm{h}}(V=0, \Delta T)-I_{\mathrm{R}}^{\mathrm{h}}(V=0,-\Delta T) \neq 0 .
$$

It hence might lead to an apparent rectification of the measured heat currents. If we assume that the gradient is distributed between the two terminals, i.e., $\Delta T=\Delta T_{\mathrm{R}}-\Delta T_{\mathrm{L}}$, there is an ambiguity in defining $-\Delta T$. For the problem of thermal rectification, the relevant situation is the one mirroring the temperature distribution: $\Delta T_{\mathrm{L}} \leftrightarrow \Delta T_{\mathrm{R}}$. This way, we get, after linearizing the currents:

$$
\delta I_{\mathrm{LR}}^{\mathrm{h}}=\left(K_{\mathrm{LL}}-K_{\mathrm{RR}}\right) \Delta T_{\mathrm{L}}+\left(K_{\mathrm{LR}}-K_{\mathrm{RL}}\right) \Delta T_{\mathrm{R}} .
$$

The second term of the right-hand side of Eq. (15) vanishes due to the fulfillment of the Onsager relations. This, however, does not apply to the diagonal coefficients, $K_{\mathrm{LL}}$ and $K_{\mathrm{RR}}$ in the first term. We will discuss below in which conditions these two coefficients become unequal in the presence of an environment, thus leading to asymmetric heat conduction.

In that case, we can define a rectification coefficient

$$
R=\left|\frac{\left(K_{\mathrm{LL}}-K_{\mathrm{RR}}\right) \Delta T_{\mathrm{L}}}{\left(K_{\mathrm{LL}}+K_{\mathrm{RR}}\right) \Delta T_{\mathrm{L}}+2 K_{\mathrm{LR}} \Delta T_{\mathrm{R}}}\right|,
$$

which reflects a thermal diode behavior for $R \approx 1$. In the particular case $\Delta T_{\mathrm{R}}=0$, it becomes

$$
R_{\mathrm{L}}=\left|\frac{K_{\mathrm{LL}}-K_{\mathrm{RR}}}{K_{\mathrm{LL}}+K_{\mathrm{RR}}}\right|,
$$

independent of the thermal gradient.

\section{Thermoelectric coefficients}

It has been discussed that asymmetries of the $L_{i j}$ coefficients might improve the thermoelectric efficiency [84]. This is the case, for instance, for broken time-reversal symmetry in the presence of a magnetic field. Then, the efficiency at maximum power depends on the ratio $L_{i j}(B) / L_{j i}(-B)$. In our device $L$ becomes asymmetric under "contact" inversion even in the absence of magnetic field.

In this case, our system acts as an engine which generates a finite power when the thermally activated current flows against a voltage gradient. Important coefficients of performance are the maximum generated power and the efficiency at maximum power. Let us specify a configuration where $T_{\mathrm{L}}>T_{\mathrm{R}}$. The extracted power

$$
P=-I_{\mathrm{L}}^{\mathrm{e}}(V) V
$$

is maximized for some drop voltage $V=V_{\mathrm{m}}$, giving

$$
V_{\mathrm{m}}=-\left(L_{\mathrm{LR}} \Delta T_{R}+L_{\mathrm{LL}} \Delta T_{\mathrm{L}}\right) /\left(2 G_{\mathrm{LR}}\right),
$$

which results in a maximum power:

$$
P_{\max }=\frac{\left(L_{\mathrm{LR}} \Delta T_{\mathrm{R}}+L_{\mathrm{LL}} \Delta T_{\mathrm{L}}\right)^{2}}{4 G_{\mathrm{LR}}} .
$$

Finally, the efficiency at maximum power $\eta_{\max }$ is easily computable from

$$
\eta_{\max }=\frac{P_{\max }}{I_{\mathrm{R}}^{\mathrm{h}}\left(V_{\mathrm{m}}\right)+P_{\max }},
$$

where one has to take into account that heat is injected from the left reservoir, $I_{\mathrm{E}}^{\mathrm{h}}$, and from the environment, $I_{\mathrm{E}}^{\mathrm{h}}$. The total injected heat is hence $-\left[I_{\mathrm{L}}^{\mathrm{h}}\left(V_{\mathrm{m}}\right)+I_{\mathrm{E}}^{\mathrm{h}}\left(V_{\mathrm{m}}\right)\right]=I_{\mathrm{R}}^{\mathrm{h}}\left(V_{\mathrm{m}}\right)+P_{\max }$.

In order to carry a more meaningful study of the setup efficiency, we also study the Carnot efficiency for this setup. In our case we need to take into account that heat is being injected by the environment, and thus, the Carnot efficiency is not simply $\eta_{C}=1-T_{\mathrm{R}} / T_{\mathrm{L}}$. We define the Carnot efficiency at the reversible point where $\frac{I_{\mathrm{L}}^{\mathrm{h}}}{T_{\mathrm{L}}}+\frac{I_{\mathrm{R}}^{\mathrm{h}}}{T_{\mathrm{R}}}+\frac{I_{\mathrm{E}}^{\mathrm{h}}}{T_{0}}=0$ (zero entropy production), which results in

$$
\eta_{C}=1+\left(\frac{I_{\mathrm{L}}^{h}}{I_{\mathrm{R}}^{h}} \frac{1-T_{0}}{T_{R}}-\frac{T_{0}}{T_{L}}\right)^{-1} .
$$

This efficiency gives a measure of the performance of our setup. 


\section{SINGLE OCCUPANCY}

We can make some analytical progress by considering a simplified situation. Let us assume the limit $E_{C} \gg k_{\mathrm{B}} T_{0}$, such that the quantum dot can be occupied by a single electron at a time. It will later help us to understand the numerical results for the general configuration presented in Sec. IV. In this case, the charge current simply reads

$$
I_{\mathrm{L}}^{\mathrm{e}}=e \frac{\Gamma_{\mathrm{L}, 0}^{-} \Gamma_{\mathrm{R}, 0}^{+}-\Gamma_{\mathrm{L}, 0}^{+} \Gamma_{\mathrm{R}, 0}^{-}}{\Gamma_{\mathrm{L}, 0}^{+}+\Gamma_{\mathrm{R}, 0}^{-}+\Gamma_{\mathrm{L}, 0}^{-}+\Gamma_{\mathrm{R}, 0}^{+}} .
$$

We consider the isoelectric case (with $V_{L}=V_{R}=0$ and $\mu_{0}=\epsilon_{\mathrm{d}}+e^{2} / 2 C$ ) and compute the linearized charge current in contact $l$ by linearizing tunneling rates as follows:

$$
\begin{gathered}
\Gamma_{l, 0}^{+}=\Gamma_{l}\left(g_{l}^{(0)}+\frac{\Delta T_{l}}{T_{0}} g_{l}^{\prime}\right), \\
\Gamma_{l, 0}^{-}=e^{\mu_{0} / k_{\mathrm{B}} T_{0}}\left(\Gamma_{l}^{+}-\frac{\Delta T_{l}}{T_{0}} \Gamma_{l} g_{l}^{(1)}\right),
\end{gathered}
$$

where we have introduced the following integrals:

$$
\begin{gathered}
g_{l}^{(n)}=\int d E\left(\frac{E+\mu_{0}}{k_{\mathrm{B}} T_{0}}\right)^{n} f^{+}\left(E+\mu_{0}\right) P_{l}(E), \\
g_{l}^{\prime n}=\int d E\left(\frac{E+\mu_{0}}{k_{\mathrm{B}} T_{0}}\right)^{n} f^{+}\left(E+\mu_{0}\right) f^{-}\left(E+\mu_{0}\right) P_{l}(E) .
\end{gathered}
$$

Note that they depend only on the corresponding terminal through the $P_{l}(E)$ function, $l=\mathrm{L}, \mathrm{R}$. In the following, we write $f^{ \pm}(E)$ for $f^{ \pm}\left(E, T_{0}\right)$.

\section{A. Seebeck asymmetry}

Linearizing Eq. (23) in terms of the expansions $(24,25)$ we obtain the Seebeck coefficients:

$$
L_{i j}=\frac{e \Gamma_{\mathrm{L}} \Gamma_{\mathrm{R}}}{T_{0} \sum_{l} \Gamma_{l} g_{l}^{(0)}} f^{-}\left(\mu_{0}\right) g_{i}^{(0)} g_{j}^{(1)} .
$$

The asymmetry in the thermoelectric coefficients, $\delta L=$ $L_{\mathrm{LR}}-L_{\mathrm{RL}}$, is hence

$$
\delta L \propto g_{\mathrm{L}}^{(0)} g_{\mathrm{R}}^{(1)}-g_{\mathrm{R}}^{(0)} g_{\mathrm{L}}^{(1)}=X_{\mathrm{RL}}^{(1)},
$$

with

$$
\begin{aligned}
X_{l l^{\prime}}^{(n)}= & \int d E d E^{\prime}\left(\frac{E+\mu_{0}}{k_{\mathrm{B}} T_{0}}\right)^{n} f^{+}\left(E+\mu_{0}\right) f^{+}\left(E^{\prime}+\mu_{0}\right) \\
& \times\left[P_{l}(E) P_{l^{\prime}}\left(E^{\prime}\right)-P_{l}\left(E^{\prime}\right) P_{l^{\prime}}(E)\right] .
\end{aligned}
$$

We can then explicitly relate the asymmetry of the Seebeck coefficients (29) to the inhomogeneous influence of the environment on the tunneling processes through each barrier, $P_{l}(E)$. This occurs when $C_{\mathrm{L}} \neq C_{\mathrm{R}}$ : both the mean and variance of the distributions become different [see Eq. 8]. Hereafter, we parametrize the asymmetry of the tunnel barrier capacitances with

$$
\kappa=\frac{C_{\mathrm{L}}-C_{\mathrm{R}}}{C} .
$$

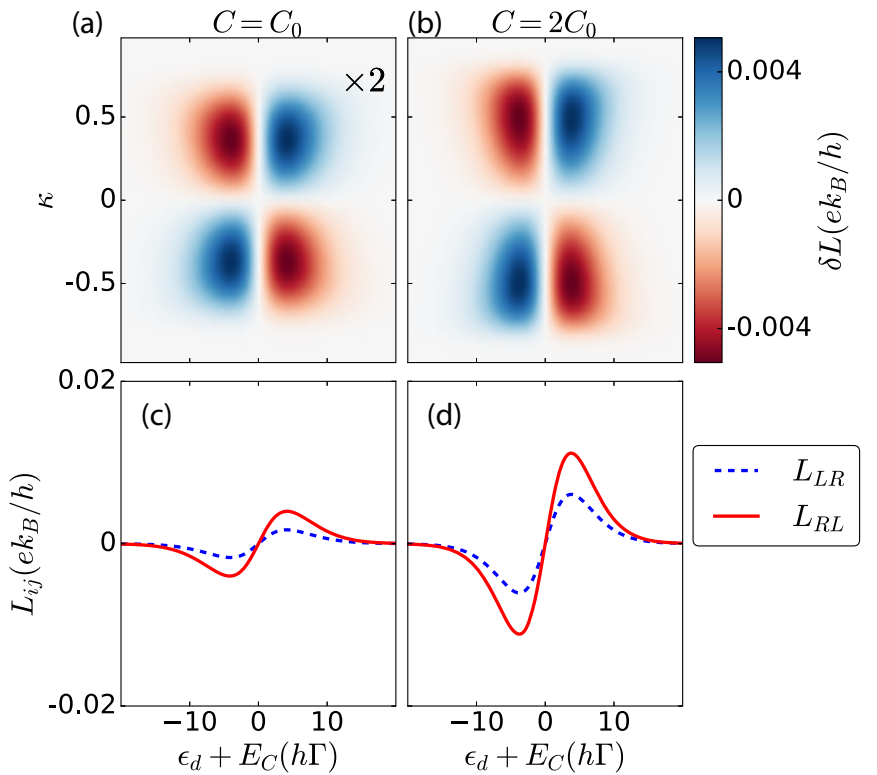

FIG. 2. Electrothermal asymmetry $\delta L$ versus dot energy-level position $\epsilon_{\mathrm{d}}+E_{C}$ and capacitance asymmetry $\kappa$ for different values of the total capacitance: (a) $C=C_{0}$ and (b) $C=2 C_{0}$. (c) and (d) show cuts of $L_{\mathrm{LR}}$ and $L_{\mathrm{RL}}$ when $\kappa=0.5$ for the values of $C$ considered in (a) and (b). We have taken $k_{B} T_{0}=2 h \Gamma_{0}$ and $\Gamma_{L}=\Gamma_{R}=\Gamma_{0} / 2$. Finite asymmetries $\kappa \neq 0$ give $\delta L \neq 0$.

Then, for a finite $\kappa$ it follows that $P_{\mathrm{L}}(E) \neq P_{\mathrm{R}}(E)$. Note that $X_{l l^{\prime}}^{(n)}$ is independent of the tunneling rates $\Gamma_{\mathrm{L}}, \Gamma_{\mathrm{R}}$.

The functions $X_{l l^{\prime}}^{(n)}$ (and therefore the asymmetry) depend on the overlapping of the Gaussian distributions $P_{\mathrm{L}}(E)$ and $P_{\mathrm{R}}(E)$, whose mean and width are, in principle, different and depend on $C_{j}$ and $C$. For very small capacitances, $C \rightarrow 0$, the two distributions are narrow, and they do not overlap. In the opposite limit, they are so wide that their difference is tiny. Optimal values of $C$ for which the effect of the environment is maximal are found in the intermediate regime.

These observations are reflected in Fig. 2. There we represent $\delta L$ for two different values of the total capacitance, $C=C_{0}$ [Fig. 2(a)] and 2 $C_{0}$ [Fig. 2(b)], with $C_{0}=e^{2} / 10 h \Gamma_{0}$, as a function of the energy level of the dot $\epsilon_{\mathrm{d}}$ and $\kappa$. We vary the symmetry of the capacitances described by $\kappa$ but not the total capacitance $C$, which is kept constant. As seen in Fig. 2, $\delta L$ is zero for $\kappa=0$. This means that an environment coupled symmetrically to both barriers is not able to break this symmetry, and therefore, inelastic scattering is not a sufficient condition for breaking this symmetry in the Onsager matrix. However, we observe that $L_{\mathrm{LR}}$ and $L_{\mathrm{RL}}$ differ for finite $\kappa$ [see Fig. 2(c) and 2(d)]. Both have the expected sawtooth line shape [47]. Remarkably, $\delta L$ changes sign when the asymmetry is inverted (i.e., when $\kappa$ changes sign) and at the particle-hole symmetry point $\epsilon_{\mathrm{d}}+E_{C}=0$, where also $L_{i j}=0$ (see Fig. 2).

The maximal value of $\delta L$ (at a finite value of $\kappa$ ) depends strongly on the total capacitance $C$. A plausible argument for such behavior is obtained by looking at the integrand of Eq. 30 that depends on the overlap of the two Gaussian functions $P_{\mathrm{L}}(E)$ and $P_{\mathrm{R}}(E)$, each one centered at energy positions that depend on $\kappa_{\mathrm{L}}^{2} E_{C}$ and $\kappa_{\mathrm{R}}^{2} E_{C}$. As discussed above, $X_{\mathrm{RL}}^{(1)}$ decreases for small enough $C$, and so does $\delta L$. 


\section{B. Thermal asymmetry}

A similar analysis is performed for the thermal coefficients. Expanding the heat rates, we obtain

$$
\begin{aligned}
& H_{l}^{+}=\Gamma_{l}\left(k_{\mathrm{B}} T g_{l}^{(1)}+g_{l}^{\prime \prime} k_{\mathrm{B}} \Delta T_{l}\right), \\
& H_{l}^{-}=e^{\mu_{0} / k_{\mathrm{B}} T}\left(H_{l}^{+}-\Gamma_{l} g_{l}^{(2)} k_{\mathrm{B}} \Delta T_{l}\right) .
\end{aligned}
$$

With these relations, we get the expression for the (diagonal) linear thermal asymmetry, $\delta K=K_{\mathrm{LL}}-K_{\mathrm{RR}}$. It can be separated into two contributions, $\delta K=\delta K_{\Gamma}+\delta K_{\kappa}$, with

$$
\begin{gathered}
\delta K_{\Gamma}=\frac{k_{\mathrm{B}} f^{-}\left(\mu_{0}\right)}{\sum_{l} \Gamma_{l} g_{l}^{(0)}}\left(\Gamma_{\mathrm{L}}^{2} \Theta_{\mathrm{L}}-\Gamma_{\mathrm{R}}^{2} \Theta_{\mathrm{R}}\right), \\
\delta K_{\kappa}=\frac{k_{\mathrm{B}} f^{-}\left(\mu_{0}\right)}{\sum_{l} \Gamma_{l} g_{l}^{(0)}} \Gamma_{\mathrm{L}} \Gamma_{\mathrm{R}} X_{\mathrm{RL}}^{(2)} .
\end{gathered}
$$

Here we have defined

$$
\Theta_{l}=\left(g_{l}^{(1)}\right)^{2}-g_{l}^{(2)} g_{l}^{(0)},
$$

which depends on the tunneling events through a single barrier. This factor is related to the thermal conductance for the energy exchange between a single terminal $l$ and the environment. Note that $\delta K_{\Gamma}$ can be finite in the case where one of the barriers is closed, $\Gamma_{j}=0$, i.e., when neither charge or energy flows through the quantum dot. This term therefore describes asymmetric energy exchange with the environment. On the other hand, $\delta K_{\kappa}$ depends on tunneling through both barriers.

We can hence distinguish two sources of rectification: $\delta K_{\Gamma}$ becomes nonzero when asymmetric tunneling barriers are considered, i.e., $\Gamma_{\mathrm{L}} \neq \Gamma_{\mathrm{R}}$, or when $\kappa \neq 0$. It relates to the different time scales that an electron stays in contact with the environment when tunneling from the left or from the right reservoir, as we discuss below. However, $\delta K_{\kappa}$ is intrinsically dependent on the dynamic coupling to the environment: it is only nonzero when the capacitances for each tunneling barrier are different.

The role of capacitance asymmetry on the heat rectification $\delta K$ is plotted in Fig. 3. There, we show $K_{\mathrm{LL}}-K_{\mathrm{RR}}$ versus the energy of the quantum dot level $\varepsilon_{\mathrm{d}}+E_{C}$ when the asymmetry in the capacitances $\kappa$ is tuned. We observe that heat rectification reverses its sign at $\kappa=0$, as expected. Importantly, we find different behaviors depending on the total capacitance $C$. For large enough $C, \delta K$ changes sign with the position of the level [see Fig. 3(b)]. This is due to a change in the relative contribution of the two terms, $\delta K_{\Gamma}$ and $\delta K_{\kappa}$, as shown in Figs. 3(e) and 3(f). This effect introduces an additional way of controlling the heat flows through the device, depending on the position of the different mean values of the $P_{j}(E)$ distributions with respect to the Fermi energy.

To assess how much the heat flows can be controlled, we explore the relative rectification $R_{\mathrm{L}}$ [see Figs. 3(c) and 3(d)]. We observe that it reaches values around $80 \%-90 \%$ at configurations where $\delta K$ is maximal [lobes in Fig. 3(a)]. The system then behaves as a thermal diode. Even larger rectifications close to $100 \%$ are reached for extreme values of the asymmetry $\kappa$. However, heat currents are almost vanishing there.

For small values of $C$, the asymmetry of the two $P(E)$ functions is large, and therefore, we find that $\delta K_{\Gamma}$ is enhanced

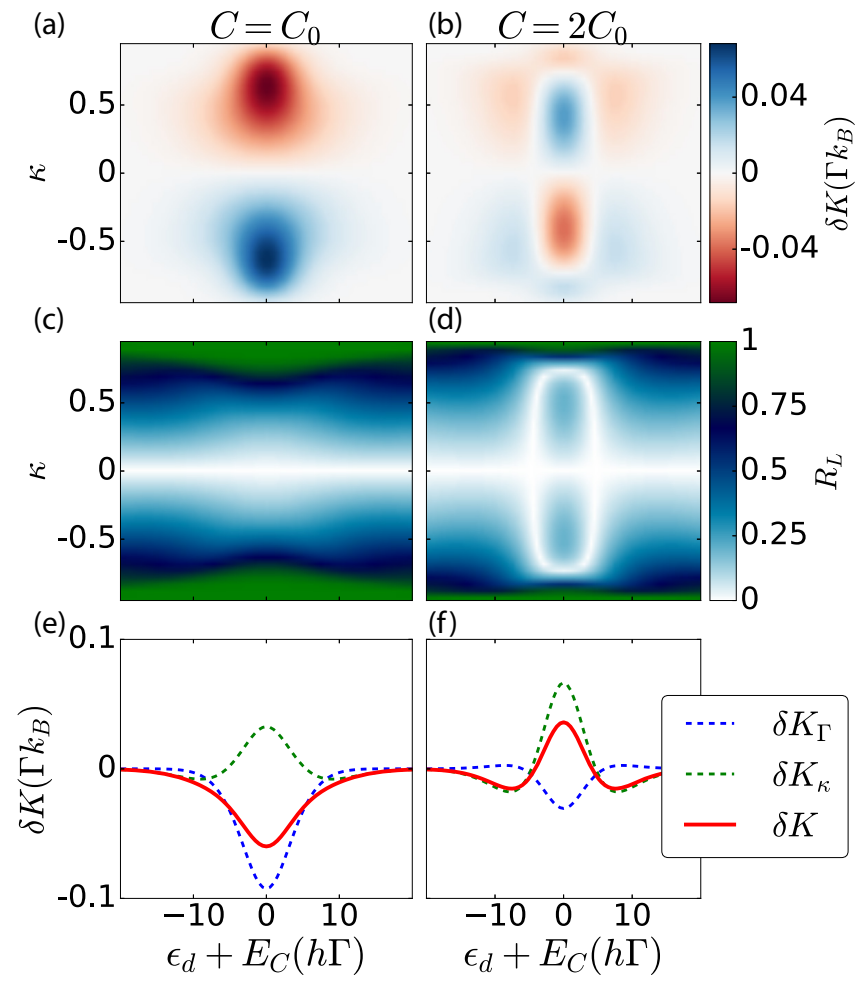

FIG. 3. Thermal asymmetry $\delta K$ versus dot energy-level position $\epsilon_{\mathrm{d}}$ and capacitance asymmetry $\kappa$ for different values of the total capacitance: (a) $C=C_{0}$ and (b) $C=2 C_{0}$. Thermal rectification occurs for $\kappa \neq 0$, i.e., $C_{\mathrm{L}} \neq C_{\mathrm{R}}$. (c) and (d) Relative thermal asymmetry $R_{\mathrm{L}}$ for the corresponding configurations in (a) and (b). (e) and (f) Total $\delta K$ (red line), tunnel $\delta K_{\Gamma}$ (dashed blue line), and capacitance $\delta K_{K}$ (dashed green line) thermal asymmetries evaluated at $\kappa=0.5$ for two different capacitances, $C=C_{0}=2 C_{0}$. The change in sign of $\delta K_{\Gamma}$, which implies the change in sign of the total $\delta K$, is clearly observed. Parameters are the same as in Fig. 2.

[and negative for $\kappa>0$; see Fig. 3(e)]. Therefore, for $C=C_{0}$, it dominates over $\delta K_{\kappa}$, which depends on the overlap $\chi_{\mathrm{LR}}^{(2)}$. Instead, for high values of $C$ we showed that $X_{\mathrm{LR}}^{(n)}$ increases, and on top of that the contribution of $\delta K_{\Gamma}$ becomes smaller [see Fig. 3(f)]. The competition between the two terms gives a nontrivial dependence of the heat rectification with $C$.

Asymmetric tunneling amplitudes also lead to a finite rectification. In this case, it depends only on $\delta K_{\Gamma}$. This is shown in Fig. 4, which plots $\delta K$ as a function of the energy of the quantum dot level $\varepsilon_{\mathrm{d}}+E_{C}$ when the asymmetry in the tunneling rate, parametrized as

$$
\lambda=\frac{\Gamma_{\mathrm{L}}-\Gamma_{\mathrm{R}}}{\Gamma},
$$

is tuned, with $\Gamma=\Gamma_{\mathrm{L}}+\Gamma_{\mathrm{R}}$. Heat rectification stems from purely different kinetic couplings. By allowing $\Gamma_{\mathrm{L}} \neq \Gamma_{\mathrm{R}}$ electrons traversing the left barrier spend shorter times in contact with the environment (and therefore, the time when energy-exchange processes are possible is shorter) than electrons at the other barrier. Therefore, environment-assisted tunneling transitions are effectively different for both junctions even though they are performed with the same environment, and $P_{\mathrm{L}}(E)=P_{\mathrm{R}}(E)$. Results for $\delta K$ at $C_{\mathrm{L}}=C_{\mathrm{R}}=C / 2$ for 


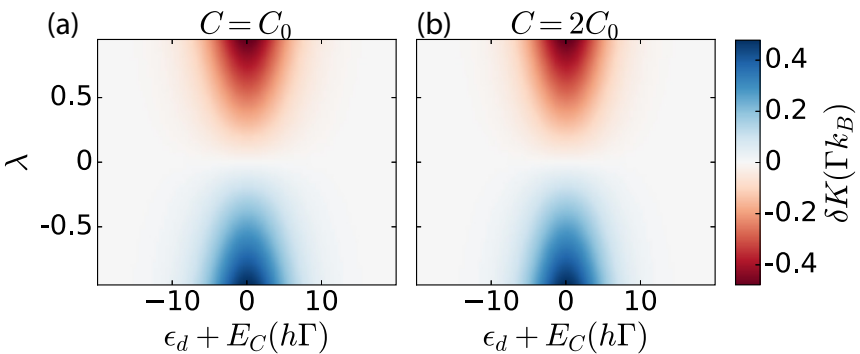

FIG. 4. Thermal asymmetry $\delta K$ versus dot energy-level position $\epsilon_{\mathrm{d}}$ and the barrier asymmetry $\lambda$ for different values of the total capacitance: (a) $C=C_{0}$ and (b) $C=2 C_{0}$. Heat rectification $\delta K \neq 0$ for $\gamma \neq 0$, i.e., $\Gamma_{\mathrm{L}} \neq \Gamma_{\mathrm{R}}$. Parameters are the same as in Fig. 2.

different values of the barrier asymmetry $\lambda$ are shown in Fig. 4. In Fig. 4 we observe that, indeed, for $\lambda \neq 0$ there is an asymmetry of the thermal coefficients which changes sign with $\lambda$. It is also observed that the shape of the asymmetry does not depend strongly on the total capacitance $C$, as expected, since the source for such heat rectification depends essentially on $\lambda$ and it does not have an electrostatic origin. Even so, the total value of the rectification decreases with the capacitance.

\section{Thermoelectric performance}

Let us focus now on how the thermoelectric performance of the device is affected by the environment. Discrete levels in quantum dot systems are known to give a high performance for being ideal energy filters. In terms of efficiency at maximum power, they reach the Curzon-Ahlborn efficiency [45], which in the linear regime is $\eta_{\mathrm{CA}}=\eta_{C} / 2$ [85]. As discussed above, energy filtering is harmed by the occurrence of inelastic scattering. Hence, the efficiency is expected to be smaller [86].

This is indeed what we observe in most of the range in Fig. 5, which shows the efficiency at maximum power $\eta_{\max }$ [Eq. 21] and the maximum power [Eq. 20] as functions of the dot gate position $\epsilon_{\mathrm{d}}$ and the asymmetry parameter $\kappa$. We find there that the system reaches efficiencies close to the $\eta_{\mathrm{CA}}$ bound or even larger [see Fig. 5(f)]. This happens for large values of the dot energy-level position and at $\kappa>0$. The efficiency is increased whenever $C_{\mathrm{L}}>C_{\mathrm{R}}$ since this coupling favors the injection of heat from the environment to the right lead, effectively helping electrons overcome the bias potential; therefore, less heat is needed from the left reservoir to extract the same power. Unfortunately, at these configurations the output power is strongly suppressed, as displayed in Fig. 5. Nevertheless, the highest $P_{\max }$ can be extracted at reasonably high efficiencies $\eta_{\max } \sim \eta_{C} / 3$ [see Fig. 5(f)]. Therefore, we observed environmentally enhanced efficiencies (compared with a perfect energy filter [45], which is bounded by the Curzon-Ahlborn limit).

We also note that the efficiency is strongly dependent on the details of the coupling to the environment, evidenced by a comparison of Figs. 5(e) and 5(f). Larger $C$ not only gives larger power; it also generates it at much larger efficiencies, compared with lower $C$. Hence, we expect that the engineering of the environmental fluctuations (by considering, e.g., nonOhmic impedances) could result in devices with enhanced thermoelectric performances.
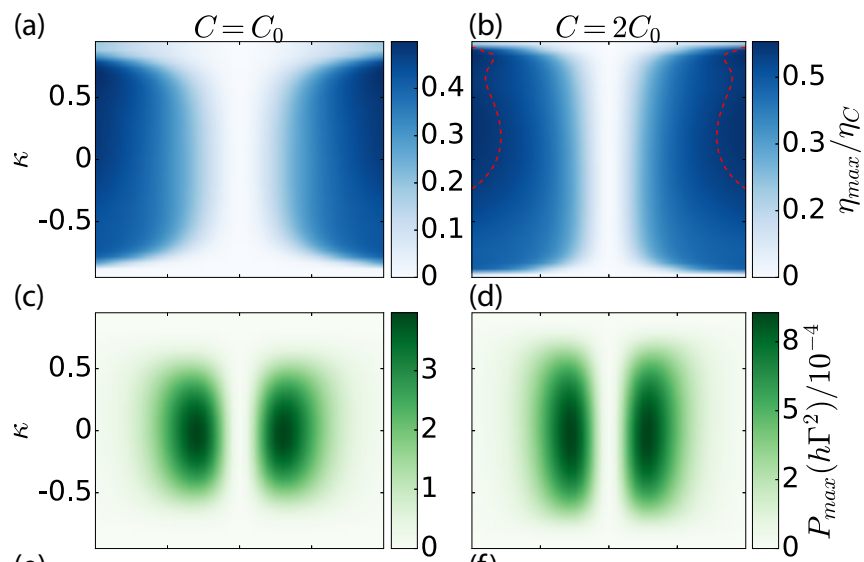

(e)

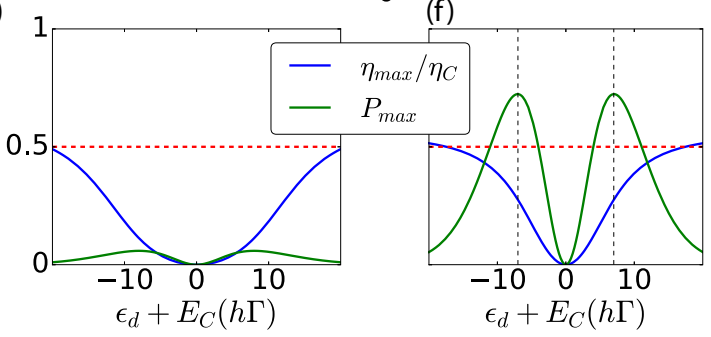

FIG. 5. Efficiency at maximum power $\eta_{\max }$ in units of the Carnot efficiency $\eta_{\mathrm{C}}$ versus the dot energy-level position $\epsilon_{\mathrm{d}}$ and the asymmetry $\kappa$ for different capacitances: (a) $C=C_{0}$ and (b) $C=2 C_{0}$. A red dashed line marks the Curzon-Ahlborn efficiency, $\eta_{\mathrm{CA}}=\eta_{\mathrm{C}} / 2$. (c) and (d) Maximum power $P_{\max }$ corresponding to the configurations shown in (a) and (b), respectively. (e) and (f) Cuts of the previous curves for $\kappa=1 / 2$ are presented for clarity. Parameters are the same as in Fig. 2.

\section{DOUBLE OCCUPANCY}

In light of the results presented in the previous section for the reduced state space with up to one electron, we come back to the general case allowing for double occupancy. That is, now we consider $|0\rangle,|\mathrm{u}\rangle,|\mathrm{d}\rangle$, and $|2\rangle$. Analytical results for the double-occupancy case are cumbersome, so we restrict ourselves to presenting our numerical simulations. The results presented in this section show the two differences: $\delta L$ and $\delta K$.

First, we present the results for $\delta L$ in Fig. 6. As expected, such difference arises only with an electrostatic asymmetry coupling, i.e., when $\kappa \neq 0$. Then, a second peak for $\delta L$ appears,
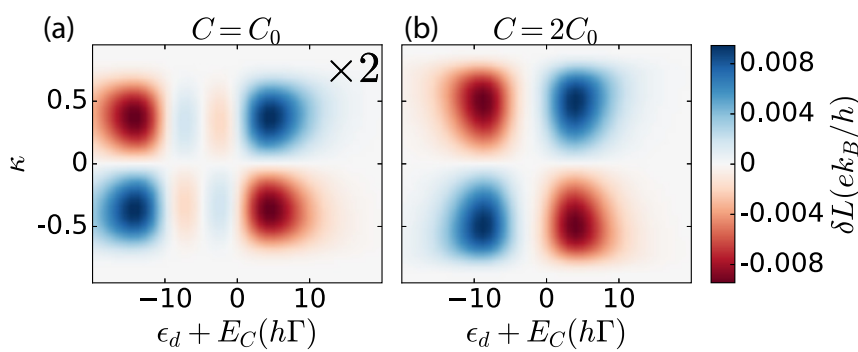

FIG. 6. Double-occupancy electrothermal asymmetry $\delta L$ versus dot energy-level position $\epsilon_{\mathrm{d}}+E_{C}$ and capacitance asymmetry $\kappa$ for different values of the total capacitance: (a) $C=C_{0}$ and (b) $C=2 C_{0}$. The crossed terms $L_{\mathrm{LR}}$ and $L_{\mathrm{RL}}$ are different for $\kappa \neq 0$, i.e., $C_{\mathrm{L}} \neq C_{\mathrm{R}}$. Parameters are the same as in Fig. 2. 

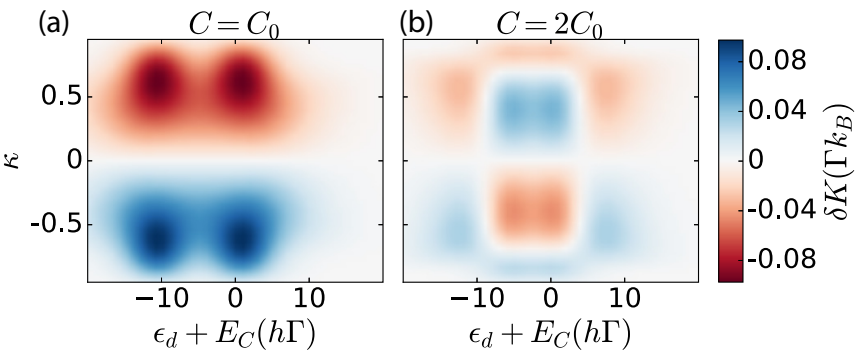

FIG. 7. Double-occupancy thermal asymmetry $K_{\mathrm{LL}}-K_{\mathrm{RR}}$ versus dot energy-level position $\epsilon_{\mathrm{d}}$ and capacitance asymmetry $\kappa$ for different values of the total capacitance: (a) $C=C_{0}$ and (b) $C=2 C_{0}$. Heat rectification $\delta K \neq 0$ for $\kappa \neq 0$, i.e., $C_{\mathrm{L}} \neq C_{\mathrm{R}}$. Parameters are the same as in Fig. 2.

corresponding to the charging of the system with a second electron. The two sawtooth oscillations are separated by the charging energy $\mu_{1}-\mu_{0}=10 h \Gamma$ [see Fig. 6(a)]. Notice that when the charging energy becomes sufficiently small, i.e., for $C=2 C_{0}$, the two features come closer, and the inner oscillations are no longer visible [Fig. 6(b)]. The behavior then resembles the one obtained for large charging energy in Sec. III.

The heat rectification for the double-occupancy case is plotted in Fig. 7. We observe the double-peak structure for a low capacitance, $C=C_{0}$, due to the two different energy levels available. As $C$ increases, there are two main effects: (i) the overall double peak tends to disappear since the charging energy diminishes, and (ii) sign changes appear in the heat rectification (for fixed $\kappa$ ) due to the greater weight of $\delta K_{\kappa}$ against $\delta K_{\Gamma}$. Therefore, the sign of the heat rectification is mainly given by $\delta K_{\kappa}$ for a large capacitance $C$, whereas $\delta K_{\Gamma}$ determines the heat rectification sign when $C$ is small.

\section{CONCLUSIONS}

In closing, we have analyzed the effect of inelasticity introduced by an electromagnetic environment on transport through a conductor (a quantum dot). We have found that even in the absence of a magnetic field, an asymmetric energy exchange with the environment can break symmetries of the transport coefficients, in particular in the thermoelectric and thermal Onsager coefficients. As a consequence an apparent rectification appears in the linear heat currents. Rectification of around $80 \%-90 \%$ is found for configurations with significant heat conduction. Furthermore, we have shown that heat injected from the environment can either improve or diminish the efficiency at maximum power output of the device when used as an engine. Efficiencies close to the Curzon-Ahlborn (or even larger) are attainable even if at vanishing power output.

We have considered here the case of a high-impedance environment. Other kinds of interactions will have different impacts on the performance of the system. The experimental ability to engineer the electromagnetic environment [87] opens the way to improve the control of thermal flows in mesoscopic conductors.

\section{ACKNOWLEDGMENTS}

We acknowledge discussions with D. Sánchez and S. Kohler. G.R. and R.L. were supported by MINECO Grant No. FIS2014-52564. R.S. acknowledges financial support from the Ministerio de Economía, Industria y Competitividad via Grants No. MAT2014-58241-P and No. FIS2015-74472-JIN (AEI/FEDER/UE). We also thank the COST Action MP1209 "Thermodynamics in the quantum regime."
[1] F. Giazotto, T. T. Heikkilä, A. Luukanen, A. M. Savin, and J. P. Pekola, Rev. Mod. Phys. 78, 217 (2006).

[2] Y. Dubi and M. Di Ventra, Rev. Mod. Phys. 83, 131 (2011).

[3] G. Benenti, G. Casati, K. Saito, and R. S. Whitney, arXiv:1608.05595.

[4] J. P. Pekola, Nat. Phys. 11, 118 (2015).

[5] R. Sánchez, B. Sothmann, and A. N. Jordan, New J. Phys. 17, 075006 (2015).

[6] P. P. Hofer and B. Sothmann, Phys. Rev. B 91, 195406 (2015).

[7] P. P. Hofer, J.-R. Souquet, and A. A. Clerk, Phys. Rev. B 93, 041418 (2016).

[8] G. Marchegiani, P. Virtanen, F. Giazotto, and M. Campisi, Phys. Rev. Appl. 6, 054014 (2016).

[9] J. Roßnagel, S. T. Dawkins, K. N. Tolazzi, O. Abah, E. Lutz, F. Schmidt-Kaler, and K. Singer, Science 352, 325 (2016).

[10] B. Karimi and J. P. Pekola, Phys. Rev. B 94, 184503 (2016).

[11] J. Meair, J. P. Bergfield, C. A. Stafford, and P. Jacquod, Phys. Rev. B 90, 035407 (2014).

[12] A. N. Jordan, B. Sothmann, R. Sánchez, and M. Büttiker, Phys. Rev. B 87, 075312 (2013).

[13] S. Donsa, S. Andergassen, and K. Held, Phys. Rev. B 89, 125103 (2014).
[14] B. Sothmann, R. Sánchez, and A. N. Jordan, Nanotechnology 26, 032001 (2015).

[15] B. Roche, P. Roulleau, T. Jullien, Y. Jompol, I. Farrer, D. A. Ritchie, and D. C. Glattli, Nat. Commun. 6, 6738 (2015).

[16] F. Hartmann, P. Pfeffer, S. Höfling, M. Kamp, and L. Worschech, Phys. Rev. Lett. 114, 146805 (2015).

[17] M. Nahum, T. M. Eiles, and J. M. Martinis, Appl. Phys. Lett. 65, 3123 (1994).

[18] M. M. Leivo, J. P. Pekola, and D. V. Averin, Appl. Phys. Lett. 68, 1996 (1996).

[19] J. R. Prance, C. G. Smith, J. P. Griffiths, S. J. Chorley, D. Anderson, G. A. C. Jones, I. Farrer, and D. A. Ritchie, Phys. Rev. Lett. 102, 146602 (2009).

[20] J. V. Koski, A. Kutvonen, I. M. Khaymovich, T. Ala-Nissila, and J. P. Pekola, Phys. Rev. Lett. 115, 260602 (2015).

[21] S. Kafanov, A. Kemppinen, Y. A. Pashkin, M. Meschke, J. S. Tsai, and J. P. Pekola, Phys. Rev. Lett. 103, 120801 (2009).

[22] T. Ruokola and T. Ojanen, Phys. Rev. B 83, 241404 (2011).

[23] D. Segal, Phys. Rev. Lett. 100, 105901 (2008).

[24] T. Ruokola, T. Ojanen, and A.-P. Jauho, Phys. Rev. B 79, 144306 (2009). 
[25] H. Thierschmann, F. Arnold, M. Mittermller, L. Maier, C. Heyn, W. Hansen, H. Buhmann, and L. W. Molenkamp, New J. Phys. 17, 113003 (2015).

[26] M. A. Sierra and D. Sánchez, Mater. Today Proc. 2, 483 (2015).

[27] K. Joulain, J. Drevillon, Y. Ezzahri, and J. Ordonez-Miranda, Phys. Rev. Lett. 116, 200601 (2016).

[28] R. Sánchez, H. Thierschmann, and L. W. Molenkamp, Phys. Rev. B 95, 241401(R) (2017).

[29] O. Entin-Wohlman, Y. Imry, and A. Aharony, Phys. Rev. B 82, 115314 (2010).

[30] R. Sánchez and M. Büttiker, Phys. Rev. B 83, 085428 (2011).

[31] J. Matthews, D. Sánchez, M. Larsson, and H. Linke, Phys. Rev. B 85, 205309 (2012).

[32] R. Sánchez, B. Sothmann, and A. N. Jordan, Phys. Rev. Lett. 114, 146801 (2015).

[33] A. A. M. Staring, L. W. Molenkamp, B. W. Alphenaar, H. van Houten, O. J. A. Buyk, M. A. A. Mabesoone, C. W. J. Beenakker, and C. T. Foxon, Europhys. Lett. 22, 57 (1993).

[34] A. Dzurak, C. Smith, M. Pepper, D. Ritchie, J. Frost, G. Jones, and D. Hasko, Solid State Commun. 87, 1145 (1993).

[35] A. S. Dzurak, C. G. Smith, C. H. W. Barnes, M. Pepper, L. Martín-Moreno, C. T. Liang, D. A. Ritchie, and G. A. C. Jones, Phys. Rev. B 55, R10197 (1997).

[36] T. E. Humphrey, R. Newbury, R. P. Taylor, and H. Linke, Phys. Rev. Lett. 89, 116801 (2002).

[37] R. Scheibner, E. G. Novik, T. Borzenko, M. König, D. Reuter, A. D. Wieck, H. Buhmann, and L. W. Molenkamp, Phys. Rev. B 75, 041301 (2007).

[38] R. Scheibner, M. Knig, D. Reuter, A. D. Wieck, C. Gould, H. Buhmann, and L. W. Molenkamp, New J. Phys. 10, 083016 (2008).

[39] S. F. Svensson, A. I. Persson, E. A. Hoffmann, N. Nakpathomkun, H. A. Nilsson, H. Q. Xu, L. Samuelson, and H. Linke, New J. Phys. 14, 033041 (2012).

[40] S. F. Svensson, E. A. Hoffmann, N. Nakpathomkun, P. M. Wu, H. Q. Xu, H. A. Nilsson, D. Sánchez, V. Kashcheyevs, and H. Linke, New J. Phys. 15, 105011 (2013).

[41] D. M. Kennes, D. Schuricht, and V. Meden, Europhys. Lett. 102, 57003 (2013).

[42] H. Thierschmann, R. Sánchez, B. Sothmann, F. Arnold, C. Heyn, W. Hansen, H. Buhmann, and L. W. Molenkamp, Nat. Nanotechnol. 10, 854 (2015).

[43] H. Thierschmann, R. Sánchez, B. Sothmann, H. Buhmann, and L. W. Molenkamp, C. R. Phys. 17, 1109 (2016).

[44] G. D. Mahan and J. O. Sofo, Proc. Natl. Acad. Sci. USA 93, 7436 (1996).

[45] M. Esposito, K. Lindenberg, and C. V. den Broeck, Europhys. Lett. 85, 60010 (2009).

[46] D. V. Averin and K. K. Likharev, J. Low Temp. Phys. 62, 345 (1986).

[47] C. W. J. Beenakker and A. A. M. Staring, Phys. Rev. B 46, 9667 (1992).

[48] M. Rey, M. Strass, S. Kohler, P. Hänggi, and F. Sols, Phys. Rev. B 76, 085337 (2007).

[49] S. Juergens, F. Haupt, M. Moskalets, and J. Splettstoesser, Phys. Rev. B 87, 245423 (2013).

[50] B. Roche, R.-P. Riwar, B. Voisin, E. Dupont-Ferrier, R. Wacquez, M. Vinet, M. Sanquer, J. Splettstoesser, and X. Jehl, Nat. Commun. 4, 1581 (2013).
[51] R. Sánchez, B. Sothmann, and A. N. Jordan, Phys. E (Amsterdam, Neth.) 75, 86 (2016).

[52] C. H. Schiegg, M. Dzierzawa, and U. Eckern, J. Phys. Condens. Matter 29, 085303 (2017).

[53] J. Argüello-Luengo, D. Sánchez, and R. López, Phys. Rev. B 91, 165431 (2015).

[54] J. H. Jiang, M. Kulkarni, D. Segal, and Y. Imry, Phys. Rev. B 92, 045309 (2015).

[55] K. Yamamoto, O. Entin-Wohlman, A. Aharony, and N. Hatano, Phys. Rev. B 94, 121402 (2016).

[56] B. Sothmann, R. Sánchez, A. N. Jordan, and M. Büttiker, Phys. Rev. B 85, 205301 (2012).

[57] B. Sothmann and M. Büttiker, Euorphys. Lett. 99, 27001 (2012).

[58] R. Sánchez, R. López, D. Sánchez, and M. Büttiker, Phys. Rev. Lett. 104, 076801 (2010).

[59] D. Bischoff, M. Eich, O. Zilberberg, C. Rssler, T. Ihn, and K. Ensslin, Nano Lett. 15, 6003 (2015).

[60] G. B. Cuetara, M. Esposito, and P. Gaspard, Phys. Rev. B 84, 165114 (2011).

[61] R. Hussein and S. Kohler, Ann. Phys. (Berlin, Ger.) 527, 610 (2015).

[62] K. Kaasbjerg and A. P. Jauho, Phys. Rev. Lett. 116, 196801 (2016).

[63] A. J. Keller, J. S. Lim, D. Sánchez, R. López, S. Amasha, J. A. Katine, H. Shtrikman, and D. Goldhaber-Gordon, Phys. Rev. Lett. 117, 066602 (2016).

[64] T. Ruokola and T. Ojanen, Phys. Rev. B 86, 035454 (2012).

[65] L. Henriet, A. N. Jordan, and K. Le Hur, Phys. Rev. B 92, 125306 (2015).

[66] P. Delsing, K. K. Likharev, L. S. Kuzmin, and T. Claeson, Phys. Rev. Lett. 63, 1180 (1989).

[67] L. J. Geerligs, V. F. Anderegg, C. A. van der Jeugd, J. Romijn, and J. E. Mooij, Europhys. Lett. 10, 79 (1989).

[68] M. H. Devoret, D. Esteve, H. Grabert, G.-L. Ingold, H. Pothier, and C. Urbina, Phys. Rev. Lett. 64, 1824 (1990).

[69] S. M. Girvin, L. I. Glazman, M. Jonson, D. R. Penn, and M. D. Stiles, Phys. Rev. Lett. 64, 3183 (1990).

[70] H. Grabert, G.-L. Ingold, M. H. Devoret, D. Estève, H. Pothier, and C. Urbina, Z. Phys. B Condens. Matter 84, 143 (1991).

[71] H. B. G. Casimir, Rev. Mod. Phys. 17, 343 (1945).

[72] H. B. Callen, Thermodynamics and an Introduction to Thermostatistics, 2nd ed. (Wiley, New York, 1985)

[73] P. Jacquod, R. S. Whitney, J. Meair, and M. Büttiker, Phys. Rev. B 86, 155118 (2012).

[74] L. Onsager, Phys. Rev. 37, 405 (1931); 38, 2265 (1931).

[75] M. Büttiker, IBM J. Res. Dev. 32, 317 (1988).

[76] P. N. Butcher, J. Phys. Condens. Matter 2, 4869 (1990).

[77] D. Sanchez and L. Serra, Phys. Rev. B 84, 201307 (2011).

[78] K. Saito, G. Benenti, G. Casati, and T. Prosen, Phys. Rev. B 84, 201306 (2011).

[79] G.-L. Ingold and Y. V. Nazarov, Single Charge Tunneling: Coulomb Blockade Phenomena in Nanostructures (Springer, Boston, 1992), pp. 21-107.

[80] C. W. J. Beenakker, Phys. Rev. B 44, 1646 (1991).

[81] J. P. Pekola and F. W. J. Hekking, Phys. Rev. Lett. 98, 210604 (2007).

[82] G.-L. Ingold, P. Wyrowski, and H. Grabert, Z. Phys. B Condens. Matter 85, 443 (1991). 
[83] S. Florens, P. Simon, S. Andergassen, and D. Feinberg, Phys. Rev. B 75, 155321 (2007).

[84] G. Benenti, K. Saito, and G. Casati, Phys. Rev. Lett. 106, 230602 (2011).

[85] F. L. Curzon and B. Ahlborn, Am. J. Phys. 43, 22 (1975).
[86] N. Nakpathomkun, H. Q. Xu, and H. Linke, Phys. Rev. B 82, 235428 (2010).

[87] C. Altimiras, O. Parlavecchio, P. Joyez, D. Vion, P. Roche, D. Esteve, and F. Portier, Phys. Rev. Lett. 112, 236803 (2014). 